\title{
Super-cool theories secure physics prize
}

Sarah Tomlin, London

Three physicists who have wrestled with the explanations behind intriguing quantum phenomena share this year's Nobel Prize in Physics.

Two Russians, Alexei Abrikosov of the Argonne National Laboratory in Illinois and Vitaly Ginzburg, the retired head of the theory group at the P. N. Lebedev Physical Institute in Moscow, are rewarded for their theoretical explanation of a form of superconductivity. Anthony Leggett of the University of Illinois at Urbana-Champaign is recognized for his work on a type of superfluid.

A superfluid forms when a fluid, such as liquid helium, effectively loses all of its viscosity and flows without any resistance. If that fluid is made up of electrons rather than atoms, the effect - electrical conductivity without resistance - is called superconductivity. Both phenomena depend upon all of the fluid's constituent atoms or 'free' electrons dropping into the same quantum state - something that happens only at low temperatures.

Superfluids are divided into more than one type of material and behaviour. They were discovered in the 1930s, when helium-4 was cooled to very low temperatures and began to flow without resistance. It was later
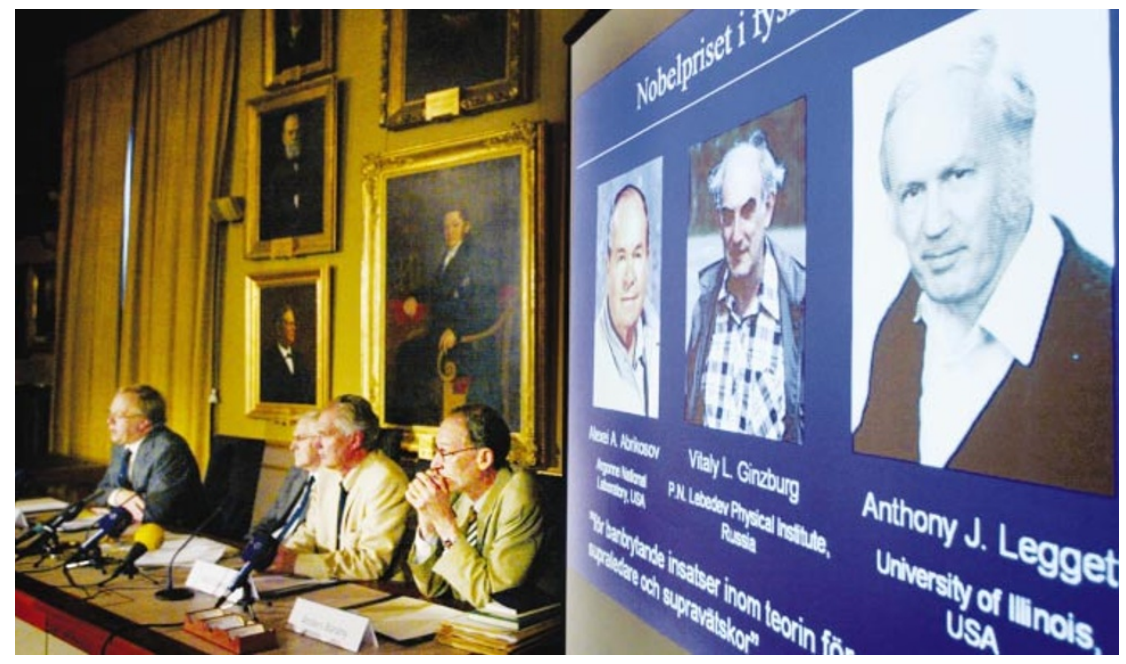

Members of the Nobel committee on physics announce this year's prize recipients.

discovered, almost by accident, that helium-3 could also become a superfluid, although at a much lower temperature — about 1,000 times lower than that needed for helium-4. This was difficult to explain, because helium3 atoms belong to a class of particles known as fermions, which don't like to share the same quantum state. It was Leggett who, in the 1970s, succeeded in explaining how this

\section{Biologists join physics preprint club}

\section{Declan Butler}

The ArXiv preprint server - physicists' favourite place for early circulation of their results - has branched out into biology.

Last month, the server's managers created q-bio, an archive for quantitative biology. The move reflects the fact that ArXiv's traditional constituency of physicists, mathematicians and computer scientists is increasingly working on biological problems, says its founder Paul Ginsparg of Cornell University in New York state.

The proportion of biology papers on ArXiv has been growing steadily, reaching $8 \%$ of the 4,500 submissions received last year. But until now, these papers have been scattered across various subdisciplines on ArXiv. The new arrangement will regroup existing content, and provide a dedicated area for quantitative biology.

Ginsparg says he hopes that the move will help to "nucleate something for newcomers" from biological disciplines. The big test, he says, will be whether biologists will follow physicists in being comfortable with circulating their 'big' papers on the archive prior to formal publication.

But papers on ArXiv are not peer-

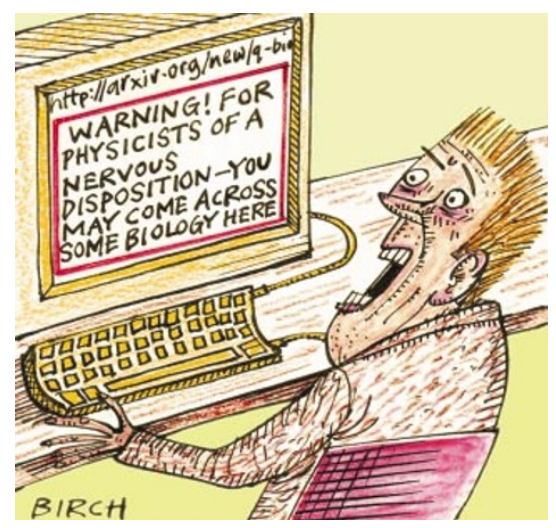

reviewed, and there is concern this could create problems if medical papers are accessed by physicians or patients. Ginsparg says he is not really worried by this, adding that the biology papers on the archive are "generally as uncontroversial as for physics".

One of the few existing biomedical preprint servers - the $B M J$ 's Netprints server - features a 'health warning' advising 'casual readers' not to act on its contents. ArXiv has no plans for such a warning, although an advisory panel does screen submissions. arxiv.org happens: the atoms first form into pairs, which then act like single particles that can occupy the same quantum state.

Superconductivity had long been known also to depend on a similar pairing of electrons. But until Abrikosov and Ginzburg's theory, building on work by a previous Nobel winner, Lev Landau, theorists struggled to explain the behaviour of a class of superconductors, called 'type II', which can remain superconducting in the presence of a magnetic field.

Their theory is now textbook material. It is particularly important for understanding the properties of superconducting magnets, such as those used in particle accelerators. "People use the Ginzburg-Landau theory all the time," says Gil Lonzarich, a physicist at the University of Cambridge, UK. "I'm looking at my board right now, and there is a Ginzburg-Landau equation on it.”

Although the three theorists share similar interests, says Lonzarich, they differ in their approach to research. Leggett is "an intuitive thinker", says Lonzarich, whereas Abrikosov is a more formal mathematician. He describes Ginzburg as one of those rare people who can do both formal and more intuitive thinking.

The three theorists helped to change the field of condensed-matter physics by recognizing the importance of interactions between atoms or electrons - the behaviour of strongly interacting particles together can be more important than individual particle behaviour at the quantum level. This "led to major changes in thinking", says Lonzarich, who argues that the recognition of the work by Abrikosov and Ginzburg, done in the 1950s, is particularly long overdue.

The Nobel chemistry prize was announced after Nature went to press. For coverage see www.nature.com/nature 